\title{
Historiografía regional y el Bicentenario de la Independencia
}

Artículos originales: HISTORIA

Recibido: 16/03/2021

Aprobado: 12/06/2021

Publicado: $14 / 10 / 2021$
Filomeno Zubieta Núñez

Universidad Nacional José Faustino Sánchez Carrión filomenozubieta@gmail.com

\begin{abstract}
RESUMEN
La conmemoración de los 200 ańos de la Independencia nacional y de vida republicana nos invita, entre otras acciones, a dar una mirada a la producción historiográfica sobre el proceso de la independencia nacional, especialmente en las provincias. ¿Qué se hizo y qué se está haciendo en las provincias y en el interior del país por conocer y valorar la participación de sus ancestros en el proceso de la independencia? Un balance sobre las publicaciones históricas relativas a la independencia de los últimos años, en esta coyuntura del bicentenario de la independencia, es lo que se ofrece en este estudio, tomando como referencia el llamado Norte Chico, el espacio geográfico que hoy ocupan las provincias de Barranca, Huaura, Huaral (la antigua provincia de Chancay) del departamento de Lima.
\end{abstract}

Palabras clave: Bicentenario, Independencia, Producción historiográfica, Norte Chico.

\section{Regional Historiography and Independence Bicentenary}

\begin{abstract}
The commemoration of 200 years of national independence as a republic invites us, among other actions, to look at the historiographic production about this process, especially in the provinces. What have we done, and what are we doing in our inland provinces to understand and recognize their ancestors' participation in the national independence process? This research offers a balanced review of historical publications related to independence from the last years. We took the so-called Norte Chico as a geographical reference area, which includes the provinces of Barranca, Huaura, Huaral (the former Chancay province) in the department of Lima.
\end{abstract}

KeYwords: Bicentenary, Independence, Historiographic production, Norte Chico. 


\section{Introducción}

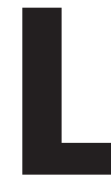

a historiografía, como estudio de la producción histórica sobre determinados temas y circunscripciones, como de las fuentes de información que se usan, de la orientación que se asigna a estos y de la recepción que tienen los autores y sus escritos, es de suma importancia.

A diferencia de los estudios de antaño en que las investigaciones sobre el Perú se realizaban desde Lima o lo ocurrido aquí se generalizaba para todo el país, en los últimos años hay una reacción positiva desde las provincias con sus intelectuales abordando temas disímiles de sus respectivas regiones. Una evidencia, por demás notable, son los estudios sobre la participación de los pueblos del interior en el proceso de la independencia del Perú.

A pesar de ser aun insuficientes y muchos de ellos desconocidos por las mayorías, estos estudios nos pueden ayudar a una valoración de la producción historiográfica relativa al proceso de la independencia ahora que estamos dentro de la coyuntura del bicentenario.

Estudios concretos sobre la producción historiográfica alusiva al proceso de la independencia son escasos, por no decir inexistentes, siendo la mayoría de estos desconocidos por el gran público lector. Hay estudios generales sobre historiografía regional que los aluden y que pueden ayudarnos a su conocimiento y valoración. Aun cuando, todos estos pertenecen a un solo autor, el suscrito, salvo el que publicaran en 1988 los sociólogos Jorge Canales Fúster y Mario Verano Conde.

En efecto, un primer registro de las publicaciones del medio se debe a los sociólogos y jóvenes docentes de la Facultad de Sociología de la universidad huachana, Jorge Canales Fuster y Mario Verano Conde que, en 1988, publican el breve libro bajo el título Guia bibliográfica de la provincia de Chancay y su área de influencia, en el que se puede constatar la ausencia de estudios sobre los pueblos de la provincia de Chancay en el proceso de la independencia.

En el 2003 como parte del libro colectivo Huacho en la historia regional está el ensayo Historiografía regional: un balance necesario que, en la sección específica sobre "Historiografía de la Independencia», se menciona a los historiadores Jesús Elías Ipinze Jordán,
Ricardo Mariátegui Oliva y Emilio Rosas Cuadros como los autores de estudios sobre esta temática.

En el año 2005 la Academia Nacional de Historia organizó su primer Congreso Nacional de Historia Regional «Pueblos, provincias y regiones en la Historia del Perú» donde presentamos nuestra ponencia Fuentes para la Historia Regional del Norte Chico que aborda sucintamente el tema de la independencia. En el 2007 la Academia Nacional de Historia publicó el libro con las principales ponencias bajo el título Pueblos, provincias y regiones en la Historia del Perú (véase las pp. 63-79).

En el 2005 publicamos la compilación de artículos de Jesús Elías Ipinze bajo el título de La antigua provincia de Chancay, siglos XVI-XVIII precedido del estudio «Jesús Elías Ipinze: trayectoria y producción historiográfica», donde se registran las decenas de sus trabajos publicados en periódicos de Lima y Huacho entre 1926 y 1959, muchos de ellos con sobre la presencia de José de San Martín y Simón Bolívar en la antigua provincia de Chancay en los días de la lucha por la independencia.

En el 2013 se publica el libro en cuyo primer capítulo se aborda el tema "Historiografía y fuentes para el conocimiento de la historia de Huacho» con un registro actualizado de los estudios sobre la participación de los pueblos de la zona en el proceso de la independencia.

A todo esto, habría que sumar algunas tesis universitarias de egresados de las universidades que, estimulados por la coyuntura del Bicentenario de la Independencia, están reseñando la temática local y valorando los antecedentes de estudios sobre la historia local y regional.

Bajo un esquema esbozado adrede, en una línea de tiempo, mostramos los estudios regionales sobre el proceso de independencia.

\section{Los primeros estudios}

Los estudios regionales sobre el proceso de la independencia a lo largo del siglo XIX son por demás escasos y en el caso del Norte Chico inexistentes, por lo menos hasta donde dan nuestras pesquisas. Ni siquiera la celebración del Centenario de la Independencia estimuló estudios sobre este tema $\mathrm{Y}$, a decir verdad, se carecían de especialistas que lo abordaran. Los 
escritores estaban dedicados al periodismo y por lo general eran maestros de educación primaria, abogados, médicos y otros profesionales que se limitaron a exaltar el heroísmo de muchos en las páginas de los periódicos locales.

El Centenario de la Independencia fue más de celebración que investigación y divulgación de lo sucedido cien ańos atrás. Pintado de fachadas, inauguración de obras, recepción de donaciones, campeonatos, concursos, desfiles, bailes, con loas a los libertadores José de San Martín y Simón Bolívar, fueron las actividades en Lima y en las principales ciudades del país. Huacho no fue la excepción, con un nutrido programa del 27 al 30 de julio. Para los propósitos de nuestro estudio es de resaltar la realización del Concurso Histórico-Literario que fue ganado por el preceptor Raimundo F. Rueda con su composición «Desembarco del generalísimo Don José de San Martín en Végueta e importancia de su labor político y militar desde el Cuartel General de Huaura para conseguir la Independencia del Perú que selló la Libertad de América del Sur».

Recién en la década del 30 del siglo XX aparecen los primeros estudios y gracias a las publicaciones de Jesús Elías Ipinze.

El historiador Jesús Elías Ipinze Jordán nacido en Sayán en 1904, con la debida formación académica en el campo de la historia en la Universidad Nacional Mayor de San Marcos, fue el primero en dedicar parte de sus investigaciones a temas de la independencia en el ámbito de la provincia de Chancay. Es más, con manejo de fuentes de primera mano, publica extensos artículos descriptivos y narrativos, por entregas, en periódicos de Huacho o de la ciudad de Lima, tal como se detalla a continuación:

1930 Síntesis de las Campañas Libertadoras en la provincia de Chancay, entre los años $1820 \mathrm{y}$ 1824. El Amigo del Pueblo. Huacho, 17 XII-1930: 1-2.

1935 Estudio que relieva la presencia de San Martín y Simón Bolívar, con un llamamiento de incorporación de estos temas en los contenidos educativos de las escuelas de su provincia.

1935 Datos sintéticos acerca de la Campaña Libertadora de Bolívar en la provincia. El Amigo del Pueblo. Huacho, 10-IV-1935: 1; 13-IV-1935: 1; 17-IV-1935: 1; 24-IV1935: 1 .
1935-36 Cronología de las actividades de Simón Bolívar en las tres oportunidades en que estuvo en la provincia de Chancay: noviembre de 1823, marzo de 1824 y diciembre de 1824 .

1936 Chancay y la Campaña Libertadora de Bolívar en 1824. El Amigo del Pueblo. Huacho, 11-V1935: 1 ; ...; 28-IX-1935: 1-2.

1936 Extenso estudio sobre los hechos de la presencia de Simón Bolívar, en la provincia de Chancay en enero de 1824, publicado en 21 ediciones de este periódico huachano.

1935-36 La Visita de Mosquera al Libertador en Pativilca. El Amigo del Pueblo. Huacho, 9-X1935: 1;...; 11-I-1936: 1-2.

Detalles de la presencia del general colombiano Joaquín Mosquera, del 12 al 15 de enero de 1824 en Pativilca, de visita al Libertador Bolívar. Este trabajo fue publicado, inicialmente, en El Comercio de Lima, el 28 de julio de 1935.

1936 La Asamblea Constituyente de Huaura de 1836.

Editorial Atlántida, S. A., Lima. 112 pp.

Único libro que publicó Ipinze, con apoyo de amigos erogantes ante la negativa de la Municipalidad Provincial de auspiciar su edición. Si bien se detalla aspectos referentes al funcionamiento del Estado NorPeruano, con sede en Huaura, durante la Confederación Perú-Boliviana; sin embargo, brinda valiosos datos sobre Huaura, Huacho y su entorno durante de décadas anteriores.

1936 Distintas capitales de la provincia de Chancay desde el Coloniaje. El Amigo del Pueblo. Huacho, 18 -IV-1936: 1; 22-IV-1936: 1; 25-IV-1936: 1.

Las que desempeñaron Chancay (Villa de Arnedo), Huaura (Villa Carrión de Velasco), Supe Viejo, Sayán y Huacho, incluyendo los años de la independencia.

1936 El Teniente Coronel don Lucas Fonseca. $\mathrm{La}$ Verdad, Huacho, 21-V-1936: 2.

Biografía del prócer de la independencia, nacido en el pueblo de Supe en 1789.

1938 Homenaje a la ciudad de Huacho. La Verdad. Huacho, 15-XI-1938: 4; 16-XI-1938: 3; $17-$ XI-1938: 2-3. 
Recuento de pasajes históricos y de personajes ilustres de Huacho, incluyendo de los años de la independencia, que fuera difundido el 10 de noviembre de 1938 por Radio Lima del Perú.

1938 Hijos Ilustres de Chancay. El Imparcial. Huacho, 11-XII-1938: 2-3.

Recuento de los personajes distinguidos en los distintos campos del quehacer intelectual, político, religioso, militar y diplomático, incluyendo la independencia. Poco antes, el 18 de diciembre de ese año, fue publicado en el diario La Prensa de Lima.

1935 Datos histórico-geográficos de Sayán. La Verdad. Huacho, 13-IV-1939: 2;14-IV-1939: 1; 15-IV-1939: 2; 17-IV-1939: 2.

Referencias desde el siglo XVI hasta su legalización como distrito en 1857, así como datos sobre sus límites, clima, producción, vías de comunicación y población actualizados.

1941 Periódicos de la provincia de Chancay desde la Iniciación de la República. El Imparcial. Huacho, 20-VI-1941: 10-11-12.

Listado de periódicos desde El Boletín del Ejército Libertador publicado en 1820, hasta el Boletin Municipal de Huaral en circulación en 1941. Artículo de homenaje a El Imparcial en su $50^{\circ}$ Aniversario.

1942 Chancay Histórico: El indio y la formación de la nacionalidad. El Amigo del Pueblo. Huacho, $1^{\circ}$-VII-1942: 1; 4-VII-1942: 1; 8-VII-1942: 1.

Charla ofrecida en la audición de Radio Mundial, el 24 de junio de 1942, organizada por el Centro Cultural Huaura.

1950 La Campańa Libertadora del General San Martín. La Prensa. Lima, 30-X-1950: 3;...; 31-XII-1950: 3.

Reconstrucción detallada del itinerario y acciones del General San Martín en la provincia de Chancay, entre el 30 de octubre y 31 de diciembre de 1820 .

1957 Datos históricos de Huacho. La Verdad. Huacho, 9-XI-1957: 2; 12-XI-1957: 2; 13XI-1957: 3.

Sinopsis histórica de Huacho desde el siglo XVI a 1874.
1966 Huacho ante la Historia. Rumbos. Huacho, 23-I-1966 ( $\left.\mathrm{N}^{\circ} 3\right)$ : 8-15.

Extractos de sus estudios históricos relevantes sobre Huacho.

José María Valega Vasallo, historiador nacido en Supe en 1887, que regentara en la Universidad Nacional Mayor de San Marcos la cátedra de Historia de la Emancipación del Perú, publicó entre 1940 y 1944 su monumental obra, hoy poco conocida y valorada, La gesta emancipadora del Perú, en 12 tomos, con algunas referencias a las ocurrencias en el Norte Chico de esos tiempos.

El Centro de Estudios Histórico-Militares, CEHMP, fundado en 1944 como institución oficial autónoma, dedicado a estudiar y difundir los acontecimientos de carácter histórico-militares del Perú contando para tal fin desde 1946 con la administración del Archivo Histórico Militar Nacional, publicó en 1948 su investigación Fecha y primer lugar del Perú donde se proclamó la independencia. En 39 páginas es el primer pronunciamiento que reconoce a Supe como el primer lugar del Perú donde se proclamó la independencia. El informe sustentatorio fue presentado por el coronel Manuel C. Bonilla, director del Archivo Histórico Militar, en la sesión del 5 de abril de 1948 del Centro de Estudios Histórico-Militares del Perú. La «Memoria del gobierno del virrey Pezuela» que señala que los supanos se declararon libres en la fecha señalada es el principal sustento; además, anexa otros documentos que aportan a afirmar la proclama aludida.

En la línea anterior el historiador limeño Ricardo Mariátegui Oliva, tomando como base un texto de historia del Perú que había publicado en 1940 donde resaltaba el hecho histórico del 5 de abril de 1819 publica en 1949 su breve libro con el título de Supe, primer pueblo del Perú que proclamó la independencia nacional, de 29 páginas. Argumenta sus asertos en los apuntes de su bisabuelo, el Dr. Francisco Javier Mariátegui, las Memorias del virrey Pezuela publicadas en 1947 y otros informes de partícipes en las gestas de 1819 y 1820 . Así, es el segundo estudio que rescata la importancia de Supe, Huacho y los pueblos del Norte Chico en la independencia.

Néstor Puertas Castro, dedicado a temas histórico-militares, publica en 1950 su estudio sobre un personaje emblemático en el Norte Chico con el título 
El general Francisco Vidal, Prócer de la Independencia Americana y Jefe Supremo de la República a través de sus Memorias (Lima: Editorial Universitas, 96 pp.) lastimosamente poco difundido y conocido.

El Instituto Sanmartiniano del Perú se funda el 23 de febrero de 1935 como institución análoga a la que dos años antes se había fundado en Argentina. Como institución patriótica de interés nacional fue reconocida por Resolución Ministerial de Educación $\mathrm{N}^{\circ} 7887$, el 16 de mayo de 1961.Entre sus miembros figuran Max Abdón Pajuelo y Emilio Rosas Cuadros, de resonancia en nuestro medio

En la década del 50 del siglo XX la vida activa al Instituto Sanmartiniano de la Provincia de Chancay, fundado el 20 de mayo de 1952 (Zubieta, 1996, p.68), bajo la presidencia del Dr. José Torres Muga se expresó en una serie de actividades de homenaje a San Martín y Huaura resaltando el hecho histórico del 27 de noviembre de 1820; esto motivó que el $\mathrm{N}^{\circ}$ 13 (julio-diciembre de 1956, 24 pp.) de la Revista del Instituto Sanmartiniano del Perú estuviera dedicado a la provincia de Chancay, tierra del general Francisco Vidal (Supe) y del coronel Andrés de los Reyes (Chancay) como son mencionados.

El Dr. Abdón Max Pajuelo, nacido en Aija (Áncash) en 1896, tuvo una larga actividad como docente, historiador y escritor, fue socio fundador del Instituto San Martiniano del Perú (1935) y socio activo de la Sociedad Geográfica de Lima. En las décadas del 30 y 40 del siglo XX publicó textos de historia universal muy acogidos en los centros educativos de educación secundaria, recibiendo reconocimientos como la Medalla de Oro por el Concejo Provincial de Lima el 28 de julio de 1942. Establecido en Huacho, como director del Colegio Luis F. Xammar, en 1965, como producto de más de 20 ańos de investigación, mientras trabajara en la Biblioteca Nacional, publica su libro El General San Martín en su Centenario (Imprenta «La Libertad», Campiña de Huacho, 221 pp.). Se trata de una segunda edición con prólogo del Dr. Cavero Egúsquiza, presidente del Instituto Sanmartiniano del Perú (la primera edición salió en 1951). Sobre el particular se publicaron encomiables comentarios y tuvo gran acogida. De éste, Isaac Salazar León nos sintetiza, «... es una enjundiosa síntesis de aspectos y acontecimientos tratados por unos y olvidados por otros, que aquí se han reunido en un todo con una finalidad de orientación» («Un li- bro y un autor", Rumbos $\mathrm{N}^{\circ} 1$, pp. 18-19). Por este libro recibió Diploma y Medalla de Oro del Concejo Provincial de Chancay, el 23 de enero de 1966, con motivo de los festejos del I Centenario de Huacho como Capital de Provincia.

\section{Bajo el manto del Sesquicentenario de la Independencia Nacional}

La celebración del Sesquicentenario de la Independencia Nacional, en el marco del gobierno militar del general Juan Velasco Alvarado, tuvo un uso político para justificar sus reformas, manejar el criterio de la independencia inconclusa, reivindicar la figura de Túpac Amaru II y los propósitos de su gobierno de completar el proceso independentista. Lo destacable fue la publicación de la Colección Documental de la Independencia del Perú en 86 tomos y 24 volúmenes de los 120 tomos y 30 volúmenes planificados, por parte de la Comisión Nacional de Sesquicentenario de la Independencia del Perú, resaltando que la independencia del Perú fue lograda por la participación de los sectores populares (en contraposición a la versión oficial del gobierno). Aquí se incluyen importantes documentos, crónicas e informes de los años de la lucha independentista, correspondientes a Huacho y las provincias de Chancay y Cajatambo, indistintamente en sus diversos tomos de utilidad para los estudios de años posteriores. En este interín La Independencia en el Perú de Heraclio Bonilla planteando la independencia concedida por los libertadores foráneos, causó resquemor natural en las esferas oficialistas.

En el ámbito regional hay que destacar algunas obras como la restauración de la Casa Bolívar en Pativilca, la inauguración del monumento a Francisco Vidal en Supe el 28 de noviembre de 1970 con la colocación de una placa: «La Nación a la gloria del general Francisco Vidal. Sesquicentenario de la Independencia del Perú. Supe, 28 de noviembre de 1970" y la puesta en funcionamiento del Museo de Sitio "Memoria San Martín» el 6 de setiembre de 1974 en la Casa-Balcón de Huaura con la develación de la placa recordatoria por el General Óscar Molina Pallochia y el Embajador de la República Argentina, Dr. Joaquín Díaz de Vivar. 
La coyuntura de la celebración del centenario de la independencia propiciaría el reflote del Instituto Sanmartiniano de la Provincia de Chancay y la fundación de sus filiales de Huaura, Végueta y otras.

Pese a toda la promoción gubernativa de la celebración del sesquicentenario, no tuvo el efecto de propiciar la producción y publicación de estudios sobre su manifestación en el ámbito regional del Norte Chico, salvo excepciones como los mencionamos a continuación.

Emilio Rosas Cuadros, nacido en el distrito de Checras (provincia de Huaura, Lima) en 1921, profesor, historiador y escritor, es uno de los referentes mayores de la historiografía regional y más en la coyuntura del sesquicentenario. Se tituló de Profesor de Educación Secundaria en la especialidad de Historia y Geografía en la Universidad Nacional Federico Villarreal con un trabajo sobre Francisco Vidal producto de sus investigaciones en archivos diversos de Lima como de Supe. Luego de una prolija revisión decidió por su publicación en 1971 con motivo del Sesquicentenario de la Independencia del Perú, bajo el título El Prócer Francisco de Vidal y su contribución a la Emancipación del Perú (Lima: Gráfica Industrial, 140 pp.). El libro fue muy bien acogido y mereció los elogios de muchos intelectuales como Gerardo Arosemena Garland, Alfonso Delboy Morales, Orestes Miranda Pacora, Jaime Chauca, Carlos Vargas, como de las autoridades municipales de Supe. Años después, en 1983, con ampliaciones producto de nuevas investigaciones, tuvo su segunda edición. En 175 páginas, con fotografías y facsímiles de documentos, es el libro más completo sobre este personaje de significativa importancia para la historia del Perú y nacido en nuestras tierras, en Supe. Entre otros, mereció el premio del Centro de Estudios Históricos-Militares del Perú.

Convencido de que sin documentos de archivos no es posible realizar trabajos de investigación histórica se esmeró en lograr el dominio de la paleografía para cuyo propósito realizó estudios especiales en la Universidad Nacional Mayor de San Marcos. Alternó el ejercicio de la docencia con las permanencias en los archivos y los encierros en su biblioteca, como la Sala de Investigaciones de la Biblioteca Nacional, Biblioteca Militar del Perú, Biblioteca del Instituto Riva Agüero, Biblioteca del Instituto Sanmartiniano del Perú, Archivo General de la Nación, Archivo
Histórico Militar del Perú, Archivo del Ministerio de Hacienda, Archivo Arzobispal de Lima, el Archivo del Seminario Santo Toribio de Lima, Biblioteca del Museo Nacional de Historia, del Archivo de la Sociedad Geográfica de Lima, de los archivos parroquiales del ámbito de la que fuera provincia de Chancay, etc.

La Provincia de Chancay en la Colonia y Emancipación (Lima, 1976) libro de 274 páginas muy bien documentado con abundantes fotografías, mapas y documentos en facsímil es otro aporte significativo del maestro Emilio Rosas Cuadros en esta coyuntura. El Dr. Fernando Gamio Palacio en el Prólogo resalta su carácter integral por abordar el proceso emancipador a lo largo de los 14 distritos de la que fuera la provincia de Chancay; pero, mucho más, lo acucioso y fundamentado de sus afirmaciones sustentados no solo en estudios serios sino y, sobre todo, en documentos de archivos, muchos de ellos desconocidos.

El general E. P. Luis Ramírez Ortiz, ilustre huachano que ocupara importantes cargos, como presidente de Obras Públicas del Ministerio de Salud, Ministro de Estado, entre otros. Con motivo de la celebración del Sesquicentenario de la Independencia Nacional, con el aporte de muchos intelectuales huachanos, reunió documentos y estudios históricos sobre la participación de los hijos de la antigua provincia de Chancay en el proceso de la independencia y publicó en 1971 con respaldo del Centro Social Chancay la obra mimeografiada titulada Antecedentes Históricos de la Provincia de Chancay: Su Participación en la Gesta Emancipadora de Nuestra Independencia.

Máximo Luna Jiménez, maestro, escritor y promotor cultural nacido en Cochamarca (hoy provincia de Oyón) muy dedicado a los pueblos de las actuales provincias de Cajatambo y Oyón, publicó una serie de ensayos históricos y literarios. El 24 de octubre de 1952 funda la Biblioteca Cajatambo y como parte de este esfuerzo inicia la circulación de Caxatambo. Revista sobre Folkore e Historia de la Provincia de Cajatambo, comprometiendo el aporte de intelectuales conocedores del Norte Chico. Se publicó hasta 1996 en que llega a su edición $N^{\circ} 5$. En sus ediciones podemos encontrar estudios sobre la presencia de fuerzas patriotas en sus tierras. 


\section{En el interregno, entre el sesquicentenario y el bicentenario de la independencia}

Entre la conmemoración del sesquicentenario y el bicentenario de la independencia nacional hay cambios significativos en la producción historiográfica regional, con estudios generales sobre algunas provincias o distritos y mayor atención al tema de la participación local y regional en el proceso de la independencia nacional.

José Arnaldo Arámbulo La Rosa, nacido en Huacho en 1943 de profesión docente con dedicación al periodismo y la investigación histórica, publicó en noviembre de 1981, en el marco del aniversario de Huacho, su libro Huacho en la historia del Perú. Una visión holística, total, de la historia de Huacho. Hasta ese momento el mejor libro sobre la historia local, por no decir el único si descontamos el breve libro de Isaías Nicho Rodríguez de 1974. Una mirada a las fuentes de información utilizadas para este libro nos brinda lo necesario para formarnos una idea de los que se tenía a disposición en la época y cómo estas fueron debidamente utilizadas. El libro en sí está dividido en seis partes. El capítulo quinto, con mayor derroche de fuentes de información, se denomina: Huacho durante la emancipación.

Alberto Carrillo Ramírez, escritor e historiador ancashino publicó en 1985 su libro Dos próceres ancashinos de la emancipación, Cayetano Requena Manuel Jesús Gonzáles (168 pp.) personajes de gravitante importancia en el proceso de la importancia. Cayetano Requena Fonseca, sacerdote, participó en la proclamación de la independencia de Supe el 5 de abril de 1819 y a partir de allí estuvo muy ligado a la historia de la independencia e inicios de la vida republicana. Igualmente, Manuel Jesús Gonzáles Grilleros, nacido en Chiquián, tuvo una larga vida política desde los tiempos de la independencia.

Carlos Zegarra Talavera, historiador autodidacta nacido en Huacho en 1932, publicó en las décadas del 80 y 90 del siglo XX, artículos históricos en diferentes medios periodísticos de Huacho como «La Verdad», «El Imparcial», "Últimas noticias», entre ellos hay algunos alusivos al proceso de la independencia en el Norte Chico resaltan: Sintesis Histórica de Huaura, Proclamación de la Independencia, Acciones de San Martín en Huaura y Huaura Cuna de la Independencia, entre otros.
Emilio Rosas Cuadros que había publicado dos libros valiosos en tiempos del sesquicentenario, presenta en 1992 su libro La Provincia de Huaura en la Colonia y Emancipación, como una segunda edición revisada y ampliada del libro dedicado a la provincia de Chancay publicado en 1976, abarcando ahora exclusivamente a los distritos y pueblos de la actual provincia de Huaura. De las muchas felicitaciones que recibió, destacamos el del Instituto Sanmartiniano del Perú. Sigue otro de sus libros más exitosos no solo por su rigurosidad sino por abordar integralmente todas las etapas de desarrollo de los pueblos de esta parte del Perú, desde tiempos remotos a la actualidad. Como algo inusual, mereció tres ediciones ya agotadas, intitulado Historia de la Provincia de Huaral: Restos Precolombinos, Coloniaje, Emancipación y República (Lima, 1993, 1994, 2005).

En esta misma línea, Emilio Rosas, en 1995 publica La Provincia de Huarochiri en la Historia, Coloniaje y Emancipación, prologado por el Dr. Gustavo Pons Muzzo que lo felicita resaltando la acuciosidad y uso de fuentes documentales en el desarrollo del estudio. Finalmente, en esta coyuntura, merece mencionarse su libro Precursores Peruanos que contribuyeron a la independencia de los Pueblos de América, (Lima, 2002, 2007), como un homenaje a los hombres que dedicaron sus energías y vidas al servicio de la causa independentista a lo largo de 341 páginas.

En este lapso publicamos, en el ańo 1991, en la revista huachana Los Especiales de Huacho que dirigía la maestra Elena Castro Malásque el artículo «Supe y la primera proclamación de la independencia» (Zubieta, Filomeno, $\mathrm{N}^{\circ} 15$, pp. 4-5) muy comentado en el medio regional y más en una fecha por demás oportuna. Tiempo después, en 1996, nuestro el libro Calendario histórico regional: Barranca-Huaura-Huaral (Huacho: Prensa Norte, 207 pp.) con breves notas por días y meses, muchas de ellas relacionadas con fechas del proceso de independencia en ámbito de lo que antaño fue el partido y luego provincia de Chancay.

Edmundo León, intelectual que trabajara en el Servicio de Estudios Rurales de Huaura, SER-Huaura, con apoyo de esta institución, publicó en 1996 el libro Huaura, villa y región: antes y después de la independencia, recogiendo un conjunto de estudios, memorias, relatos y crónicas de inicios del siglo XIX que aluden a la importancia que tuvo Huaura en el interregno de la independencia e inicios de la vida republicana. 
En este período aparecen algunos libros sobre historia local abordando el tema de la independencia, así sea brevemente. El profesor Arturo Vergara Manrique en 1996 publico su libro Paramonga en la historia; Guillermo Mongrut Steane en ese mismo año nos ofrece la segunda edición de libro Ensayo histórico de la provincia de Barranca; Pedro Castañeda Pardo, por su lado, en el 2005, publica su texto Conociendo la provincia de Huaral.

Manuel Ildauro De los Santos Camones, maestro de profesión nacido en Végueta en 1919, recogiendo la versiones orales de antiguos pobladores de su tierra natal, siempre resaltó la importancia de Végueta como lugar de desembarco de don José de San Martín y su expedición libertadora el 12 de noviembre de 1820 , reuniendo todos estos testimonios y con sus propias reflexiones publica en 1999 su libro Végueta, Autentico lugar del desembarco de la expedición Libertadora, 1820-1999 (años atrás había circulado una edición restringida a mimeógrafo). Es más, gracias a sus gestiones como de las autoridades del lugar, el Congreso de la República del Perú declaró a Végueta por Ley 23942 Distrito Histórico de la Independencia Nacional, el 12 de octubre de 1984.

Domingo Torero Fernández de Córdova, intelectual y promotor cultural huachano (1929-2011), como lector empedernido, hurgó en nuestro pasado $y$, como muchos autodidactas, fue un historiador muy informado y escribió muchos artículos que publicó en revistas especializadas del medio. En el libro Historia de Huacho que editara Iván Hoyos en 1999 fue considerado su estudio Huacho, Puerto de Libertad que valora la importancia de Huacho en el proceso de la independencia, especialmente en el desembarco de Lord Cochrane y José de San Martín, con todas las derivaciones consiguientes.

En el libro colectivo Huacho en la historia regional publicado en el año 2003 por los docentes de ciencias sociales de la Facultad de Educación de la universidad huachana, está el ensayo Huacho en la Independencia del docente Elifio Carrera Huaranga quien analiza la participación de Huacho dentro del contexto internacional y americano en que se da proceso de la independencia.

Miguel Ángel Silva Esquén, docente universitario y promotor cultural por excelencia, también entre sus publicaciones le ha dedicado atención al proceso de independencia. Ya en el 2004 publica su artículo
«¿Desde cuándo el Perú es libre e independiente?», en la revista Mirador Regional (Huacho, abril, $\mathrm{N}^{\circ} 8$, p. 17). A este se suman sus artículos en la revista del Patronato de Defensa del Patrimonio Cultural del Valle de Huaura y Ámbar.

En el año 2008 el Obispado de Huacho cumplió sus 50 años de creación y funcionamiento, con ese motivo se publicó su libro conmemorativo Diócesis de Huacho: una iglesia joven con una rica historia. 50 años de vida pastoral (Obispado de Huacho, 438 pp.) con estudios de connotados intelectuales resaltando el rol de la iglesia católica no solo en los últimos 50 años del obispado sino a lo largo de su presencia desde la colonización española. Entre los estudios se incluye uno intitulado Huacho y la Iglesia en la independencia del Perú del historiador y docente sanmarquino Raúl Adanaqué Velásquez. Con documentos hallados, entre los expedientes del Archivo del Obispado en formación, reconstruye pasajes del comportamiento de la iglesia huachana con el proceso de la independencia, como del pueblo en general. Concluye que «fue en Norte chico donde se mostró la mayor participación popular por la independencia». (Adanaqué, 2008, p. 213).

\section{La coyuntura del Bicentenario de la Independencia}

Los actos de celebración o conmemoración de los 200 años de nuestra independencia como de trayectoria republicana se han iniciado tardíamente en el Perú. Mientras los países vecinos ya la celebraron (Argentina, Chile, Ecuador y otros) nosotros recién lo estamos haciendo y culminará en 2021 y 2024.

Los primeros actos conmemorativos o celebratorios no partieron de los entes gubernativos sino de los académicos, como la Universidad Nacional Mayor de San Marcos o el Instituto Sanmartiniano del Perú, por citar a dos de los más significativos.

La Universidad Nacional Mayor de San Marcos, haciéndose eco de lo que ocurría en países vecinos asume la iniciativa de desarrollar un amplio programa de actividades para revalorar el proceso de la independencia a la luz de los 200 años, a través de su Vicerrectorado de Investigación con la Dra. Aurora Marrou Roldán al frente; proyectan: congresos, proyectos multidisciplinarios, premios y publicaciones. 
Las actividades se inician el 30 de abril del 2010 con el I Congreso hacia el Bicentenario. 200 años de vida republicana. Balance y perspectivas con presentación de diez conferencias magistrales y ponencias de académicos de historia, literatura, derecho, medicina humana, veterinaria, biología, filosofía e ingeniería metalúrgica, Se plantearon los objetivos siguientes:

1. Iniciar las actividades por el Bicentenario de nuestra independencia.

2. Promover investigaciones sobre el período de la emancipación en la etapa histórica de las luchas por la independencia en sus dimensiones política, militar y económica, en el Perú y América Latina.

3. Propiciar estudios que presenten un balance de lo logrado en las distintas disciplinas científicas y académicas en el Perú y en especial en San Marcos a lo largo de 200 ańos de vida republicana.

4. Poner en debate con carácter prospectivo los retos a futuro tanto para las distintas disciplinas académica y científicas como para el país en general.

A lo largo de los 10 años transcurridos son muchas las actividades desarrolladas y el balance de sus logros escapan a este breve informe.

Desde las esferas gubernativas la reacción fue tardía. Recién el 6 de octubre de 2016, el gobierno del Presidente Pedro Pablo Kuczynski expidió la Resolución Suprema No 246-2016-PCM² conformando la Comisión Multisectorial de naturaleza temporal encargada de formular la Agenda de Conmemoración del Bicentenario de la Independencia del Perú, con el encargo de:

a. Proponer la agenda de conmemoración del Poder Ejecutivo;

b. Proponer y supervisar concursos de ideas y proyectos múltiples que permitan celebrar la conmemoración a escala regional y nacional y;

c. Supervisar los concursos nacionales a celebrarse para definir el logotipo, los audiovisuales y las canciones que identificarán la conmemoración, entre otros.

El 6 de junio de 2018, se publica el decreto supremo que crea el Proyecto Especial Bicentenario de la Independencia del Perú que llevara a cabo la formulación de la Agenda de la conmemoración, puesta en marcha el 10 de noviembre de 2018, con el lanzamiento de la Agenda Bicentenario en Ayacucho y simultáneamente en 22 Regiones del país.

Por otro lado, con fecha 20 de octubre de 2016, el Congreso de la República aprobó la conformación de la Comisión Especial Multipartidaria Conmemorativa del Bicentenario de la Independencia del Perú. Esta comisión acordó, entre otras actividades: promover investigaciones, publicaciones y conversatorios sobre tres hechos importantes: la Independencia nacional (1821), el establecimiento del Primer Congreso Constituyente del Perú (1822), y la conmemoración de las batallas de Junín y Ayacucho (1824). Publica su revista digital Rumbo al Bicentenario.

En el caso de los pueblos del llamado Norte Chico, en Huacho y Huaura, se iniciaron algunas actividades tempranamente gracias a la iniciativa del maestro Alberto Magno Collantes Osorio miembro del Instituto Sanmartiniano del Perú y de los directivos del Instituto Sanmartiniano, filial Huaura, que insistieron mediante documentos y visitas ante las dependencias públicas la designación de comisiones de bicentenario que programen las actividades celebratorias para toda la década.

Así la Universidad Nacional José Faustino Sánchez Carrión por Resolución Rectoral Nº 04143 del 25 de enero del 2013 designa la Comisión Universitaria de Sensibilización por el Bicentenario de la Independencia de la Provincia de Huaura, bajo la presidencia del Dr. Filomeno Zubieta Núnéz. Por su lado la Municipalidad Provincial de Huaura-Huacho, en paralelo, designó una Comisión pero fue recién a inicios de junio del 2015 en que se instaló oficialmente con la denominación de Comisión Provincial del Bicentenario de la Independencia del Perú, con la participación de representantes de las instituciones culturales y académica del medio (Instituto Sanmartiniano, filial Huaura, Patronato de Defensa del Patrimonio Cultural del Valle Huaura y Ámbar, Ínsula Huacho, Universidad Nacional José Faustino Sánchez Carrión, entre otras), la misma que ha venido desarrollado importantes actividades bajo la presidencia de don Carlos Bisso Drago.

Lo más destacable de la labor de las comisiones de bicentenario fue la realización de conversatorios, mesas redondas y conferencias sobre la participación 
de los pueblos del Norte Chico en el proceso de la independencia nacional. Aun cuando hasta ahora se está privilegiando los estudios alrededor de la presencia de San Martín en nuestro medio, desdeñando o postergando para después el estudio de la participación de Simón Bolívar y del propio José Faustino Sánchez Carrión en este proceso y de construcción del Perú republicano.

La Comisión de Celebración del Centenario del Nacimiento del Historiador Jesús Elías Ipinze formado en el año 2003 «con el propósito de rescatar, valorar y divulgar la producción literaria e historiográfica del Dr. Jesús Elías Ipinze Jordán con motivo del Centenario de su nacimiento", publicó cinco libros del aludido historiador, dos libros dedicados a la independencia, justo cuando se iniciaban las acciones celebratorias del bicentenario de la independencia. Uno en el 2010, La campaña libertadora del general San Martín en el Norte Chico (144 pp.) y el otro en el 2011 La campaña libertadora de Bolivar en la provincia de Chancay, 1823-1824 (132 pp.), el primero bajo la responsabilidad de Freddy Gutiérrez Zelvaggio y el segundo de Filomeno Zubieta Núñez. Se trata del acopio del conjunto de sus artículos publicados entre 1930 y 1950 en los periódicos de Huacho y Lima sobre la presencia de San Martín y Simón Bolívar en la circunscripción de la antigua provincia de Chancay y convertidos en libros de los referidos títulos.

Los docentes de la Universidad Nacional José Faustino Sánchez Carrión Filomeno Zubieta Núńez, Manuel Benza Pflucker y Augusto Escalante Apaéstegui (recientemente fallecido) en el año 2010 publicaron el libro José Faustino Sánchez Carrión: legado y vigencia (Huacho: Gráfica Imagen. 120 pp.) buscando que el personaje sea conocido y valorado por la comunidad universitaria. Se resaltan aspectos como su semblanza biográfica, su rol como ideólogo y político de la independencia, así como su faceta literaria. En anexos se incluyen sus cartas firmadas como El Solitario de Sayán, así como los referidos a la universidad faustiniana.

El profesor e historiador Alberto Collantes Osorio (nacido en Végueta), miembro del Instituto Sanmartiniano del Perú y autor de muchos libros de historia peruana y de la independencia, publicó en el 2011 su libro Misión educadora de don José de San Martin (Lima: Editorial San Marcos. 128 pp.) con la finalidad de exaltar y puntualizar la valiosa obra educativa de don José de San Martín. Este texto fue un acicate a otros a emprender similar labor.

José Fernández Sánchez, maestro-historiador nacido en Huacho, autor de muchísimas publicaciones de historia, algunas de ellas sobre el entorno del Norte Chico, es junto a Alberto Collantes Osorio uno de los primeros en incentivar el interés por la investigación de la historia regional vinculado a los 200 ańos de la independencia nacional. De él tenemos muchos estudios dedicados a los docentes y estudiantes del ámbito regional, preferentemente. Ya en el 2006 publica Selección de lecturas sobre la independencia del Perú como parte de los materiales educativos para estudiantes de secundaria con apoyo del Instituto Superior Pedagógico Privado América; poco después, aparece su trabajo Hechos memorables durante la guerra de Independencia del Perú también para estudiantes del nivel secundario con apoyo de Ediciones Ugartinas.

En el 2010 publica El Norte Chico y el Bicentenario de la Independencia Politica en el Perú (Huacho: Gráfica Imagen. 60 pp.); en el 2012 participa en el III Congreso Internacional «Hacia el Bicentenario de la Independencia 200 años de vida Republicana» que organiza la UNMSM con su ponencia Guerra de la Independencia 1818-1824. Presencia de los pueblos del Norte Chico. Esta ponencia con los agregados da lugar a la publicación en el 2013 del libro Presencia de los pueblos del Norte Chico en la guerra de la Independencia, 1819-1824 (Huacho: Gráfica Imagen. 108 pp.); en el 2014 edita Juan Francisco Vidal y la Hoz. Prócer, patriota y héroe popular por la Independencia en el Perú como parte del Laboratorio Pedagógico de Ciencias Histórico-Sociales que promueve con Ínsula-Huacho.

A todas estas publicaciones, y otras no mencionadas del profesor José Fernández, se agrega su reciente libro del 2020 Ensayos sobre la Independencia. El pueblo y sus líderes en su Serie: Laboratorio Pedagógico de Ciencias Histórico-Sociales, cuya presentación nos honró su redacción.

El profesor Eleazar Otilio Vivar Espinoza, autor de muchas publicaciones sobre la provincia de Cajatambo, en el 2013 dio a conocer su libro Evolución histórica de la demarcación política de la provincia de Cajatambo (Lima: Gráfica Quinteros, 189 pp.). Todo el libro, sustentado en documentos y buena bibliografía, gira alrededor de las idas y venidas 
de esta antigua provincia de Cajatambo en los años del proceso de la independencia y de los inicios de la República, siendo por lo mismo importante para valorar el comportamiento de sus hijos en el proceso de la independencia.

Filomeno Zubieta Núñez, como docente de la Universidad Nacional José Faustino Sánchez Carrión ha publicado artículos, libros y participado en eventos donde, también, dedicó parte de sus esfuerzos al tema de la participación de los pueblos del Norte Chico en la independencia nacional. En el 2013 publicó su libro Huacho: historia, cultura e identidad (Huacho: Gráfica Imagen, 560 pp.) donde le dedica parte de su tercer capítulo a esta temática bajo el título El desembarco de San Martín en Huacho donde, luego de contextualizar, trata sobre el recibimiento a Lord Cochrane en 1819, el desembarco de San Martín en Huacho el 10 de noviembre de 1820, San Martín en Huaura y el significado de su presencia en Huacho y Huaura.

José Faustino Sánchez Carrión y la identidad Faustiniana es el estudio que publica en el 2018 pretendiendo establecer el nivel de valoración del personaje por los estamentos de la universidad que lleva su nombre (revista Big Bang Faustiniano, Vol. 7, N ${ }^{\circ}$ 4, pp. 21-24). En el 2019 a petición de la Comisión de Bicentenario del Congreso de la República se preparó un breve estudio intitulado "Cochrane y San Martin en el Norte Chico: desembarco y proclamas independentistas publicado» en la revista Rumbo al Bicentenario ( $\mathrm{N}^{\circ}$ 5, pp. 5-6). Los días 5, 6 y 7 de noviembre del 2019 la Pontifica Universidad Católica, PUCP, organizó el XI Coloquio Internacional Hacia el Bicentenario de la Independencia del Perú donde participa con la ponencia intitulada, «La presencia de Lord Cochrane en el Norte Chico y sus implicancias: la primera proclamación de la independencia en Supe, 5 de abril de 1819».

El hoy extinto profesor universitario César La Cruz Salvador y otros colegas (Sergio La Cruz Orbe, Óscar Yanapa Zenteno, Pascual Cornejo y Roberto Loza) reflexionan sobre El valor de la mentira en la historia politica del Perú en los 200 años de la independencia en un artículo publicado en el 2014 en la revista Big Bang Faustiniano (Vol 3, No 2, pp. 28-31).

José Jorge Nava Pittaluga, periodista huaurino integrante del Instituto Sanmartiniano del Perú, ha dedicado los últimos años a la publicación de compendios con estudios que resaltan la importancia del Huaura y el Norte Chico en el proceso de la independencia. Mencionamos: Honor y gloria a Huaura y al general José de San Martín editado en el 2017; Villa de Huaura, cuna de la independencia del Perú publicado en el 2018; y el compendio general de todo lo que había reunido bajo el sugerente título de José de San Martín Libertador del Perú. Huaura Cuna de la Independencia $y$ de la Campana de la Libertad (año 2019), con un recorrido desde Atahualpa a San Martín.

Luis Alberto Rosado, profesor e historiador huachano, es uno de los jóvenes escritores que, en los últimos años, le ha dedicado mayor atención al tema de la participación de los pueblos del Norte Chico a la independencia nacional sea en publicaciones, participación en certámenes o divulgación a través de las redes sociales. Algunos de sus trabajos son los mencionamos: En el año 2012 participa en el V Congreso Nacional de Historia desarrollado en la Universidad Nacional Mayor de San Marcos con la ponencia Del cabildo de indios al municipio independiente: parcialidades indigenas y conflictos de poder en el pueblo San Bartolomé de Huacho, 1808-1822. (Lima: UNMSM, 19 pp.). En el 2013 da a conocer su estudio El Norte Chico y el Bicentenario. Recuperado de http:// es.slideshare.net/slideshow/embed_code/22273992.

En el 2014 participa en el Concurso Narra la independencia desde tu pueblo organizado por el Congreso de la República y ocupa el primer puesto entre 60 participantes con su trabajo Infieles al rey: El pueblo de San Bartolomé de Huacho durante el proceso de la independencia, 1812-1822 que en el 2017 es publicado junto a otros trabajos en el libro Narra la independencia desde tu pueblo: Huacho, Arequipa, Tarapacá [Estensoro, Juan Carlos \& Méndez, Cecilia, editores. Lima: IFEA-IEP, pp. 59-104. En el 2018 se publica su estudio El partido de Chancay: población, migración y economía a inicios del siglo XIX en el libro colectivo Doscientos años de artes, letras y vida cotidiana en el norte del Perú. [Arrizabalaga, Carlos, Pérez, Crisanto \& Cortez, Shirley, editores]. Lima: pp. 139-165).

La revista digital Rumbo al Bicentenario $\left(\mathrm{N}^{\circ} 5\right.$, pp. 10-13) del Congreso de la República incluye el artículo de Luis Rosado, "Huacho, el pueblo que manifestó los primeros actos de patriotismo y ofrendó a la causa de la independencia cuatro mártires en 1819». En este mismo año (2019) su estudio «Supe: el primer pueblo que proclamó su independencia. 
Economía regional, sociedad, relaciones de parentesco, espías y conspiradores» es incluido en el compendio que editaron los historiadores Daniel Morán y Carlos Carcelén Las Guerras de la independencia entre dosfuegos. Cambios y permanencias (Trujillo: Editorial de la Universidad Nacional de Trujillo, pp. 177-202). En el 2020 su ponencia «iLa revolución traicionada?: indígenas forasteros y originarios en el proceso de independencia, Huacho, 1819-1835" es programada para el Congreso Internacional «1820, La Ilusión de la Libertad. El liberalismo revolucionario en España y América en los años veinte que debió desarrollarse en Las Cabezas de San Juan y Sevilla (España) los días 10, 11 y 12 de marzo 2020.

En lo que va del ańo, signada por las limitaciones impuestas por la pandemia, Luis Rosado se ha mantenido muy activo participando en cuanta actividad ha sido invitado, por lo que el listado de los temas desarrollados es numeroso. Es de destacar su participación en las actividades organizadas por Dirección Regional de Comercio Exterior y Turismo del Gobierno Regional de Lima; igualmente en el IX Congreso Nacional de Historia organizado por la Facultad de Ciencias Sociales de la UNMSM con la ponencia Don José de San Martín y el histórico balcón de Huaura: de promesa a proclamación de la Independencia del Perú. Es más, a través de su página «Micro historias del Norte Chico por Luis Rosado», contiene la información de los eventos en los que participa de utilidad para consulta de estudiantes de todos los niveles como de los interesados.

Bruno Gutiérrez Torero, intelectual y promotor cultura huachano, está dedicado a temas de la independencia los que los expone en charlas y conferencias resaltando la condición de Huacho como cuna de la independencia y, además, tiene muchas publicaciones de las que resaltamos la edición de varios números de la revista El Social con temas sobre la historia de Huacho; así en el $\mathrm{N}^{\circ} 1$ correspondiente a setiembre del 2015 está el artículo «Huacho, puerto de libertad» (pp. 14-15), igualmente "La casa del balcón de Huaura» (p. 22). Otro artículo con el título «Huacho, puerto de libertad» fue publicado en la revista digital del Congreso de la República Rumbo al Bicentenario (2019, N ${ }^{\circ}$, pp. 13-16). En el 2019 publica en formato de folleto Huacho, puerto de libertad. Desembarco de las Escuadras Libertadoras en Huacho (1819-1829) (Huacho: Gráfica Rimey. 40 pp.). En los títulos de sus publicaciones sigue los pasos de su tío Domingo Torero Fernández de Córdova. En los últimos meses ha participado en varios eventos virtuales sobre la temática de la independencia.

Christian Sánchez Jara, docente de la especialidad de ciencias sociales que desempeńa su magisterio en Barranca, cuenta con muchas investigaciones dedicadas al proceso de la independencia. En el año 2010 resultó ganador del «I Concurso hacia el Bicentenario de la Independencia del Perú», organizado por la Pontificia Universidad Católica del Perú, la Academia Nacional de Historia y la Organización de Estados Iberoamericanos (OEI), estudio que luego fue publicado con el título Hacia el Bicentenario de la Independencia del Perú 2021 por el Ministerio de Educación, la Organización de Estados Iberoamericanos y la Pontificia Universidad Católica del Perú en el 2011. En el año 2014 publica su estudio Los Afrosoldados del Norte Chico y su participación en la Independencia del Perú.

Más adelante, en el 2018, en el libro colectivo Las Guerras de la Independencia en Clave Bicentenario. Problemas y posibilidades, bajo responsabilidad de Daniel Morán y Carlos Carcelén, se incorpora su trabajo «Unidos por la libertad: afro soldados del Río de la Plata, y Perú en la guerra independentista del norte chico (1819-1825)». En el 2019 con motivo del sesquicentenario de la independencia proclamada en Supe, Christian Sánchez Jara, da a conocer su estudio ¿Y el sector popular dónde estaba? La participación del bajo pueblo en la proclama de la independentista de Supe: 5 de abril de 1819. En el 2019 da a conocer su artículo «Huaura y Barranca en la coyuntura independentista. Opiniones de diversos viajeros extranjeros bajo la coyuntura del proceso de la independencia del Perú» (véase: http://barranca.pe/las-provinciasde-huaura-y-barranca-vista-por-los-viajeros-en-lacoyuntura-independentista-1815-1824/).

En lo que va del año, a través de los recursos virtuales, se mantiene muy activo dando a conocer los resultados de sus investigaciones con temas sobre el proceso independentista. Estuvo en el IX Congreso Nacional de Historia organizado por la Facultad de Ciencias Sociales de la UNMSM con la ponencia Las independencias de los pueblos de Supe y Barranca en el contexto 1817-1820. Administra la página Bicentenario de los pueblos de Barranca, Supe y Pativilca, 1819-1824 que va alimentando en forma 
permanente con libros y recursos educativos referidos a este tema; a este se suma su blog: https://christiandelahistoriaysusensenanzas.blogspot.com/

Edgar Pérez Piñán, docente investigador de la especialidad de ciencias sociales coautor con Christian Sánchez Jara y Jhonny Briceño Mejía del libro Apuntes históricos de la provincia de Barranca. Tiene trabajos sobre la participación de los pueblos de Supe, Barranca y Pativilca en el proceso de la independencia. En el año 2010 publica su estudio «Participación de Barranca durante el proceso de independencia» en la revista Quillca (Ańo 1, Nº 1, p. 2) que alienta como docente de la Facultad de Educación, UNJFSC. Con Carmen Mc Evoy y Christian Sánchez Jara participó en el Conversatorio Magistral sobre Revalorización e Identidad de la Efemérides de Supe (04 de abril 2019) con el tema "Contribución del pueblo de Barranca a la independencia peruana y su significado histórico a propósito del centenario de la proclamación de la independencia en Supe el 05 de abril de 1819», organizado por la Municipalidad Provincial de Barranca.

En el Fórum-panel: Barranca en la gesta independentista del Perú, con motivo de la conmemoración de los 198 años de la independencia del Perú, Edgar Pérez, participó con la ponencia «Contribución económica del pueblo de Barranca a la independencia peruana». A estos y otros estudios agregamos: «Trascendencia del epistolario de Bolívar durante su estancia en Pativilca (1824-1825)» y «La gesta del patriota supano Juan Francisco de Vidal La Hoz y su aporte a la educación actual», en proceso de edición. A igual que su coterráneo Christian Sánchez Jara, en este 2020, está dando a conocer sus investigaciones mediante conferencias virtuales. Es más, con otros intelectuales e interesados en investigar, dar a conocer y resaltar la participación de los pueblos de la hoy provincia de Barranca en el proceso de la independencia fundaron y mantienen en plena actividad a la Asociación Cultural Bicentenario de la Independencia - Provincia de Barranca, Bicentenario Acubiproba.

Pieter van Dalen Luna, arqueólogo de profesión y docente de la Universidad Nacional Mayor de San Marcos, no es ajeno a los temas históricos relacionados con la independencia que, por su residencia en Huaral, le ha dedicado estudios a su entorno. En el 2016 se publica su ponencia «El estado del patrimonio cultural en la costa norcentral peruana hacia el bicentenario» presentado en el VI Congreso Internacional. Hacia el
Bicentenario de la Independencia. 200 años de vida republicana (pp. 295-340). En este 2016 publica su libro La provincia de Huaral en la historia cuyo último capítulo «Proceso histórico de la provincia de Huaral en la República» da unas pinceladas a la participación de los hijos de esta provincia en el proceso independentista (Lima: Gutenberg editores, 144 pp.).

Un panorama que ofrecía la doctrina de Iguarí en el contexto de las luchas por la independencia brinda su artículo "Análisis de un censo eclesiástico de la Doctrina de Iguarí de 1821 (provincia de Huaral)» publicado el año 2011 en la revista Guara del Museo de Arqueología de la UNJFSC ( $\mathrm{N}^{\circ} 10$, pp. 15-20). En el año 2017, publica su artículo «El distrito de Pacaraos durante la independencia nacional» en el Boletín de Lima ( $\mathrm{N}^{\circ} 190$, pp. 37-46) con una mirada general desde sus orígenes y registro de sus sitios arqueológicos, pasando por la ocupación colonial y deteniéndose en su participación en el proceso de independencia; investigación que había sido presentada en el VII Congreso Internacional. Hacia el Bicentenario de la Independencia (1821.2021) desarrollado en el 2017 y publicada en junio del 2019 en su libro de ponencias, pp. 343-362.

En el 2020, Pieter van Dalen publica su estudio documentado «La comunidad campesina de San Martín de Maní (provincia de Oyón) durante la colonia y la independencia nacional: situación y aportes» en el Boletín de Lima una de las pocas investigaciones sobre la jurisdicción del antiguo Cajatambo, hoy provincia de Oyón.

La historiadora argentina Silvia Verónica Escanilla Huerta, docente en la Universidad San Andrés de Buenos Aires, ha dedicado muchos de sus estudios al proceso de la independencia en el Norte Chico. Mencionamos algunos: en el año 2013 participa en la XIV Jornadas Interescuelas de Historia en Mendoza (Argentina) con su estudio De la lealtad a la negociación de la obediencia. Transformaciones politicas durante la guerra de independencia en el Perú. El caso del pueblo de Huacho (1820-1822). Al siguiente año, 2014, publica su estudio «La transformación política de la sociedad virreinal. El norte chico frente a la guerra de Independencia (1820-1822)» en la Revista Documenta Delaware Historia Militar (No. 4, Lima, Perú, agosto 2014). En el 2018 su trabajo "Patriotas de su propia tierra. La costa central norte en el contexto de las incursiones de Cochrane, 
1819» es incluido en el libro colectivo Las guerras de Independencia en clave bicentenario. Problemas y posibilidades editado por Daniel Morán, Carlos Carcelén (Lima: Grupo Gráfico del Piero).

Luis Hoces López, intelectual y promotor cultural huachano, desde la Municipalidad Distrital de Huaura y luego desde la Municipalidad Provincial estuvo y está alentando conversatorios y debates sobre la contribución de los pueblos del Norte Chico en el proceso de la independencia.

Ricardo Bustamante Abad, docente de ciencias sociales en la Facultad de Educación de la Universidad Nacional José Faustino Sánchez Carrión, actual presidente de la Comisión de Celebración de Bicentenario de la Independencia de esta Universidad, publicó en el 2011 su libro Huacho 12000 años de historia (Gráfica Rimey SRL, 264 pp.) con una mirada panorámica del entorno local donde están aspectos relacionados con el proceso de la independencia. En el 2016 publicó el artículo «A propósito del Bicentenario de la Independencia Participación del Pueblo de la Región Lima - Provincias en la Independencia Nacional» en coautoría con Carmen Pablo Agama en la revista Big Bang Faustiniano ( ${ }^{\circ} 5$, pp. 5-10).

En el 2017, Ricardo Bustamante con los docentes Carmen Pablo Agama, Manuel Changana y Riss García Grimaldo, publica "A propósito del bicentenario de la independencia nacional. Papel de los corsarios, piratas y mercenarios en la independencia del Perú», también en la revista Big Bang Faustiniano (Vol. 6, No 3, pp. 14-18). Al que se suman sus disertaciones en marco de las actividades de la Comisión Bicentenario que preside. Aquí resaltamos, por ejemplo, el ciclo de conferencias bajo el epígrafe: La independencia en la memoria colectiva de los pueblos de Huacho y la región Lima y la reafirmación de nuestra identidad histórica, local, regional y nacional, del 05 de setiembre al 31 de octubre del 2020.

La Dirección Regional de Comercio Exterior y Turismo del Gobierno Regional de Lima que dirige el Mg. José Antonio Espinoza Huertas, de manera especial desde que se iniciaron las medidas restrictivas con la pandemia del Covid-19, ha realizado eventos académicos virtuales, resaltando el rol de los pueblos de la actual Región Lima en el proceso de la independencia, comprometiendo la participación de los intelectuales locales, nacionales e internacionales. Merece destacarse el Ciclo de conferencias Ruta de
Conmemoraciones Bicentenario de la Independencia del Perú en la Región Lima 2020, con un nutrido programa de webinars como de foros conmemorativos en Huaura, Barranca, Huaral, Cajatambo, Canta, Cañete, Huarochirí y Yauyos, entre los meses de agosto a diciembre del 2020.

En este breve ensayo historiográfico se ha resaltado la atención concitada por el general Francisco de Vidal La Hoz por parte de los estudiosos, tanto en anteriores como en este último tramo. El libro de Néstor Puertas Castro publicado en 1950, El general Francisco Vidal, Prócer de la Independencia Americana y Jefe Supremo de la República a través de sus memorias. Años más tarde, el de Emilio Rosas Cuadros que, en 1971 y luego en 1983, en una edición ampliada y documentada, había publicado El Prócer Francisco de Vidal y su contribución a la Emancipación del Perú (Lima, 175 pp.) y en el 2014 el breve libro del maestro José Fernández Sánchez Juan Francisco Vidal y la Hoz. Prócer, patriota y héroe popular por la Independencia en el Perú.

En el 2019 coronel de caballería EP Enrique Gargurevich Godoy, presidente del Instituto de Estudios Históricos del Ejército, publica Juan Francisco Vidal y La Hoz, Primer Soldado del Perú (Lima: IEHE, 136 pp.) estudio en 6 capítulos y 5 anexos con documentos y que lo presentó en el mismo Supe el 2 de abril del 2019 con motivo de la celebración del Bicentenario de la Proclamación de la Independencia del 5 de abril de 1819.

Es más, el 12 de julio del 2019, con algunas personalidades y muchos integrantes del Instituto de Estudios Históricos del Ejército, el coronel Enrique Gargurevich, funda la Orden de la Legión General Vidal, "para difundir el legado del Primer Soldado del Perú a nivel nacional e internacional, para que este prohombre de la Patria sea conocido por las nuevas generaciones", siendo electo su primer presidente activo el referido coronel Enrique Gargurevich y el coronel EP Jorge Barrantes como presidente nato. Y, a inicios del año $2020 \mathrm{el} \mathrm{coronel}^{\circledR}$ EP Jorge Barrantes Arrese (asesor del Instituto de Estudios Históricos del Ejército), en coautoría del historiador Luis Guzmán Palomino, publica el libro Francisco de Vidal, la memoria de El Republicano tomando como referencia principal a las Memorias del general Vidal.

Como las acciones conmemorativas del Bicentenario de la Independencia continuarán por lo 
menos hasta el 2024, es de esperar que nuevos estudios se irán sumando a lo reseñado hasta aquí. Ya tendremos oportunidad de un balance general.

\section{Conclusiones}

a. Para nuestro estudio la historiografía es una herramienta útil que nos permite identificar, conocer y valorar la historia de la historia de la participación de los pueblos del Norte Chico en la independencia, los autores de los estudios, las fuentes de información utilizadas, la orientación que tienen estos estudios y la recepción que tuvieron.

b. Los primeros estudios sobre la independencia en el Norte Chico si bien son escasos sí son relevantes. Están conformados por los estudios del Jesús Elías Ipinze Jordán publicados por entregas en los periódicos de Huacho y Lima destacando la presencia de las expediciones de José de San Martín y Simón Bolívar en el ámbito de la provincia de Chancay. Se suman estudios del Centro de Estudios Histórico-Militares (1948) y del historiador Ricardo Mariátegui Oliva (1949) que resaltan la importancia de Supe como el primer lugar del Perú donde se proclamó la independencia, el 5 de abril de 1819.

c. La celebración del Sesquicentenario de la Independencia Nacional, a pesar de toda la promoción desde las esperas oficialistas del Gobierno Militar, no tuvo el efecto de propiciar estudios y publicaciones sobre su expresión en el ámbito regional, salvo breves notas periodísticas. Sí hay que resaltar los dos trabajos del historiador Emilio Rosas Cuadros, uno de corte biográfico sobre el general Francisco Vidal Laos $(1971,1983)$ y otro sobre la provincia de Chancay durante la colonia y la emancipación (1976).

d. El interregno de la conmemoración del sesquicentenario y el bicentenario de la independencia y la vida republicana en la producción y publicación de estudios relativos a la participación de los pueblos del Norte Chico en la independencia se distingue fundamentalmente por la aparición de libros integrales sobre determinados distritos donde el tema de la independencia ocupa un hito significativo. Son los casos de Huacho en la historia del Perú
(1981) de Arnaldo Arámbulo la Rosa, Historia de la provincia de Huaral: Restos precolombinos, coloniaje, emancipación y república (Lima, 1993, 1994, 2005) y La provincia de Huarochiri en la Historia, coloniaje y emancipación (1995) de Emilio Rosas Cuadros, Paramonga en la historia (1996) de Arturo Vergara Manrique, Calendario histórico regional: Barranca-Huaral-Huaura (1996) de Filomeno Zubieta Núnez, Ensayo histórico de la provincia de Barranca (1996) de Guillermo Mongrut Steane, Conociendo la provincia de Huaral (2005) de Pedro Castañeda Pardo. A esto se suman algunos compendios Historia de Huacho (1999) que editara Iván Hoyos Méndes, Huacho en la historia regional (2003) publicación de los docentes de la Facultad de Educación de la Universidad de Huacho o libro conmemorativo Diócesis de Huacho: una iglesia joven con una rica historia. 50 años de vida pastoral (2008) del Obispado de Huacho.

e. La conmemoración de los 200 años de la independencia y de vida republicana en el Norte Chico se expresa en una prolífica producción historiográfica sobre el proceso de la independencia, la diversificación del uso de las fuentes de información con lo que ofrecen repositorios como el Archivo del Obispado y el Archivo Regional de Lima, el surgimiento de nuevos estudiosos en sus provincias facilitando su dedicación y compromiso, investigaciones reflexivas a diferencia de lapsos anteriores que eran mayormente narrativos, presencia de estudios específicos para pueblos como Huacho, Chancay, Huaral, Barranca, Supe, Pativilca, entre otros. Es de esperar investigaciones sobre la presencia de Simón Bolívar como la influencia de José Faustino Sánchez Carrión en la afirmación de la independencia y la construcción del Perú republicano.

f. A 200 años de la independencia y de nuestra vida republicana, desde la perspectiva de la producción historiográfica, tenemos el reto de realizar estudios no solo de lo ocurrido en el proceso de la independencia sino, también, valorar el derrotero de los pueblos a lo largo de estos 200 ańos. ¿Qué lecciones nos dejó la independencia y qué tanto lo aprovechamos para ser diferentes y mejores? ¿Qué hemos hecho a lo largo de los 200 años como pueblos conformantes del Norte Chico? El reto, la tarea, sigue pendiente. 


\section{Referencias}

Adanaqué, Raúl (2008). Huacho y la iglesia en la Independencia. Diócesis de Huacho. Una iglesia joven con una rica historia. 50 años de servicio pastoral. Huacho: Gráfica Imagen, 440 pp.

Aguirre, Carlos (2018). ¿La segunda liberación? El nacionalismo militar y la conmemoración del sesquicentenario de la independencia peruana. La revolución peculiar. Repensando el gobierno militar de Velasco. Lima: IEP ediciones, pp. 41-70.

Arámbulo, Arnaldo (1982). Huacho en la historia del Perú. Lima, Perú: Edit. Rapid Print. 167 pp.

Asociación Cultural Bicentenario de la Independencia - Provincia de Barranca, Bicentenario Acubiproba. https://www.facebook.com/bicentenario.acubiproba.5

Bicentenario de los pueblos de Barranca, Supe y Pativilca, 1819-1824. https://www.facebook.com/ groups/280664145384278

Canales, Jorge y Verano, Mario (1988). Guia bibliográfca de la provincia de Chancay y su área de influencia. Huacho: Editorial Gráfica Bisso. 102 pp.

Centro de Estudios Histórico-Militares, CEHMP (1948). Fecha y primer lugar del Perú donde se proclamó la independencia. Lima, Perú: Miranda. 39 pp.

Cochrane, Thomás (1917). Memorias de Lord Cochrane, Conde de Dundonald de Lord Cochrane. Caracas: Editorial América. 301 pp.

Collantes, Alberto (2011). Misión educadora de don José de San Martín. Lima, Perú: Editorial San Marcos. 128 pp.

Dirección Regional de Comercio Exterior y Turismo del Gobierno Regional de Lima. https://www.facebook. com/dirceturlimaoficial

Fernández, José (2013). Presencia de los pueblos del Norte Chico en la guerra de la Independencia, 1819-1924. Huacho, Perú: Gráfica Imagen. 108 pp.

Ipinze, Jesús (2010). La campaña libertadora del general San Martín en el Norte Chico. Huacho, Perú: Comisión de Celebración del Centenario del Nacimiento de Jesús Elías Ipinze. 144 pp

Ipinze, Jesús (2011). La campaña libertadora de Bolivar en la provincia de Chancay, 1823-1824. Huacho: Comisión de Celebración del Centenario del Nacimiento de Jesús Elías Ipinze. 132 pp.

Mariátegui, Ricardo (1949). Supe, primer pueblo del Perú que proclamó la independencia nacional. Lima, Perú: Patria. 29 pp.
Morán, Daniel \& Carcelén, Carlos (editores, 2018). Las Guerras de Independencia en Clave Bicentenario. Problemas y posibilidades. Lima: Grupo Gráfico del Piero. 203 pp.

Morán, Daniel \& Carcelén, Carlos (editores, 2019). Las guerras de independencia entre dos fuegos. Cambios y permanencias. Editorial de la Universidad Nacional de Trujillo. 236 pp.

Nicho, Isaías (1976). Historia de Huacho, 1874-1974. Sintesis y otras notas. Huacho, Perú:

Pezuela, Joaquín de la (1947). Memoria del Gobierno del Virrey Pezuela. Madrid, Espańa: Escuela de Estudios Hispanoamericanos. 912 pp.

Rosas, Emilio (1976). La provincia de Chancay en la colonia y la emancipación. Lima: Editorial Industrial. 274 pp.

Rosa, Emilio (1983). El prócer Francisco de Vidal y su contribución a la emancipación del Perú. Segunda edición. Lima, Perú: Editorial Industrial. 175 pp.

Rosado, Luis (2020). Micro historias del Norte Chico. https://www.facebook.com/search/top?q=micro $\% 20$ historias $\% 20$ del $\% 20$ norte $\% 20$ chico $\% 20$ por $\% 20$ luis\%20a.\%20rosado

Sánchez, Christian (2020). Bicentenario de los pueblos de Barranca, Supe y Pativilca, 1819-1824. https://www.facebook.com/groups/280664145384278

Vivar, Eleazar (2013). Evolución histórica de la demarcación politica de la provincia de Cajatambo. Lima: Gráfica Quinteros. 189 pp.

Zubieta, Filomeno (1991). Supe y la primera proclamación de la independencia. Los Especiales de Huacho, (15), pp. 4-5.

Zubieta, Filomeno (1996). Calendario histórico regional: Barranca-Huaura-Huaral. Huacho, Perú: Ediciones Prensa Norte. 208 pp.

Zubieta, Filomeno (2003). Historiografía regional: un balance necesario. Huacho en la historia regional. Huacho, Perú: UNJFSC, pp.7 - 29.

Zubieta, Filomeno (2007). Fuentes para la historia del Norte Chico. En Academia Nacional de la Historia (Ed.), Pueblos, provincias y regiones en la Historia del Perú (p. 63-79). Lima, Perú.

Zubieta, Filomeno (2013). Huacho: Historia, Cultura e Identidad. Huacho: Gráfica Imagen. 560 pp

Zubieta, Filomeno; Benza, Manuel; Escalante, Augusto (2010). José Faustino Sánchez Carrión: legado y vigencia. Huacho: Gráfica Imagen. 120 pp. 